\title{
Maximising resource utilisation in scheduling of underground mining works with multiple faces
}

\author{
D Cortes Codelco, Chile \\ Y Martínez Codelco, Chile \\ M Silva Züblin, Chile
}

\begin{abstract}
Scheduling of underground mining activities is based on the correct allocation of resources to ensure the fulfilment of the required physical advance of the project. In the case of mining tunnel construction, the scheduling of activities is based on three aspects - availability of required services of each unit operation (power, ventilation, industrial water, compressed air and drainage), availability of resources required (equipment and mining crew), and availability of faces for physical allocation and resource utilisation. In block caving mining projects, the development involved, particularly in the production level, is associated with multiple faces, which makes the allocation of resources complex compared to operations with isolated faces where resources are captive.

Given the multiple face scenario, a scheduling model was generated based on the integration of the availability of the three aspects previously mentioned - faces, resources and services. These parameters are integrated in basic productive units (BPU), corresponding to entities defined by a certain quantity of available faces associated to a requirement of services, equipment and mining crew. These entities result in a rate of 300 to $350 \mathrm{~m}$ of mine tunnel development by month by each BPU.

This model was developed and applied in the Chuquicamata Underground Project. The application of the model in mine scheduling allowed an increase of performance of the development by $78 \%$ per month in its first month of implementation instead of the $17 \%$ increase extrapolated from the trend to that date, allowing to reach a rate in range of 1,700 to $1,800 \mathrm{~m}$ per month.
\end{abstract}

Keywords: resource allocation, mine tunnel construction, block caving, Chuquicamata Underground Project

\section{Introduction}

In the mining industry, the development of underground mines implies the scheduling of all the activities related to mining work to achieve the construction milestone in time. In this kind of work, mine tunnel development is critical to production and operation of the mine. For this, scheduling in accordance with strategic objectives is key to the success of underground mining projects and operations.

Depending on the tunnel's intended use and the project area, the development area might be advanced with single or multiple faces with different specifications as required. Where a single development face exists, the focus is on maximising the face development rate which typically results in not maximising the utilisation of resources (equipment and mining crews). This scenario results in more expensive costs per metre of development and development rates of 230-240 m per month. On the other hand, in areas where multiple development faces are available, both optimising and maximising the resource utilisation and development metres result in a lower per metre development cost, since all resources are utilised and doing productive work, and the monthly development rate per face is $40-60 \mathrm{~m}$ per month.

A simple methodology is proposed to schedule monthly development activities in order to maximise utilisation of resources when the strategy corresponds to a multiple face advance. The proposed approach defines a minimum requirement in terms of services (specifically the minimum of ventilation and power), 
resources (equipment and mining crews), and mining faces. This approach defines basic productive units (BPU) equivalent to independent working areas that ensures maximisation of resources and an advance rate of 300-350 m per month.

It is important to note that resource allocation scenarios with multiple options, resources, interactions and constraints is complex and simulation approaches have been applied, as found in mining literature (Greberg \& Sundqvist 2011; Salama \& Greberg 2012; Wolgram et al. 2012). This approach presents a more simplified methodology that maximises utilisation of resources. The outcome of this methodology is simple implementation which can be updated based on monthly blasting rates. The monthly drill-and-blast metres depend on the optimisation of the process, which is not considered in this study.

\section{Methodology}

A BPU is a working area with a given number of faces that works as an independent or semi-independent system (power and ventilation) that, through the incorporation of specific resources (inputs), results in a specific quantity of blasting (outputs). Resources interact in a specific way and order in the development cycle that generates blasting and, therefore, metres of advance as its final result.

The number of metres advanced depends on the drilling and blasting efficiency. For this case study, a deliverable of $3.6 \mathrm{~m}$ of tunnel development is considered for each development blast.

Resources $\rightarrow$ Face $\rightarrow$ Development cycle $\rightarrow$ Blasting $\rightarrow 3.6 \mathrm{~m}$ of tunnel development

Figure 1 describes the unit operations that define the development cycle. This sequence is applied to each allocated development face, which provides the physical resource to allocate equipment and crews to achieve the cycle.

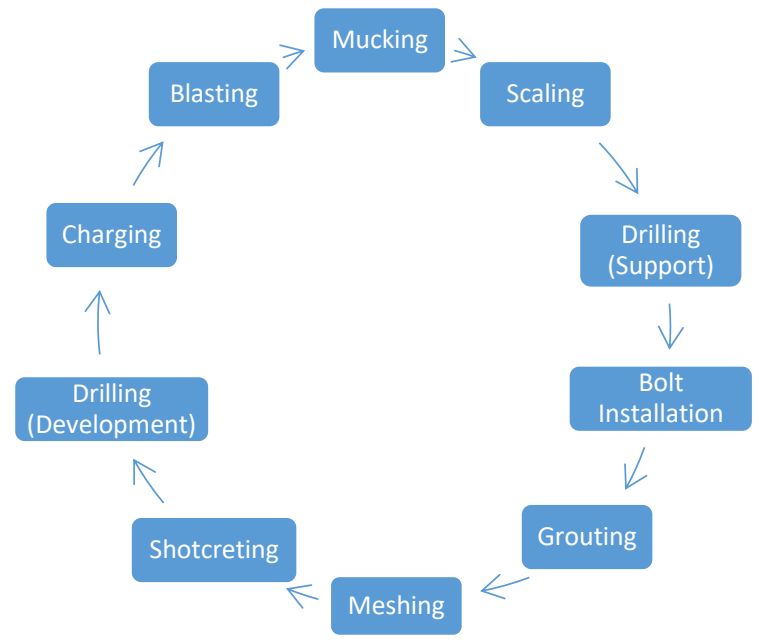

Figure 1 Development cycle in underground mines

In a multiple face context, the availability of development faces is not the bottleneck, but the availability and allocation of resources (equipment and crews), and maximising utilisation through scheduling is the prime objective. This target is achieved by introducing the model of the BPU.

A BPU corresponds to a set of five main faces and four auxiliary faces. Main faces are being developed simultaneously by one of the resource activities of the development cycle (Figure 2). In the case that any of the main faces are not available (scheduled or non-scheduled conflict), the resources are re-allocated to an auxiliary face to ensure maximisation of utilisation of that resource (auxiliary face minimises the idle resource). 


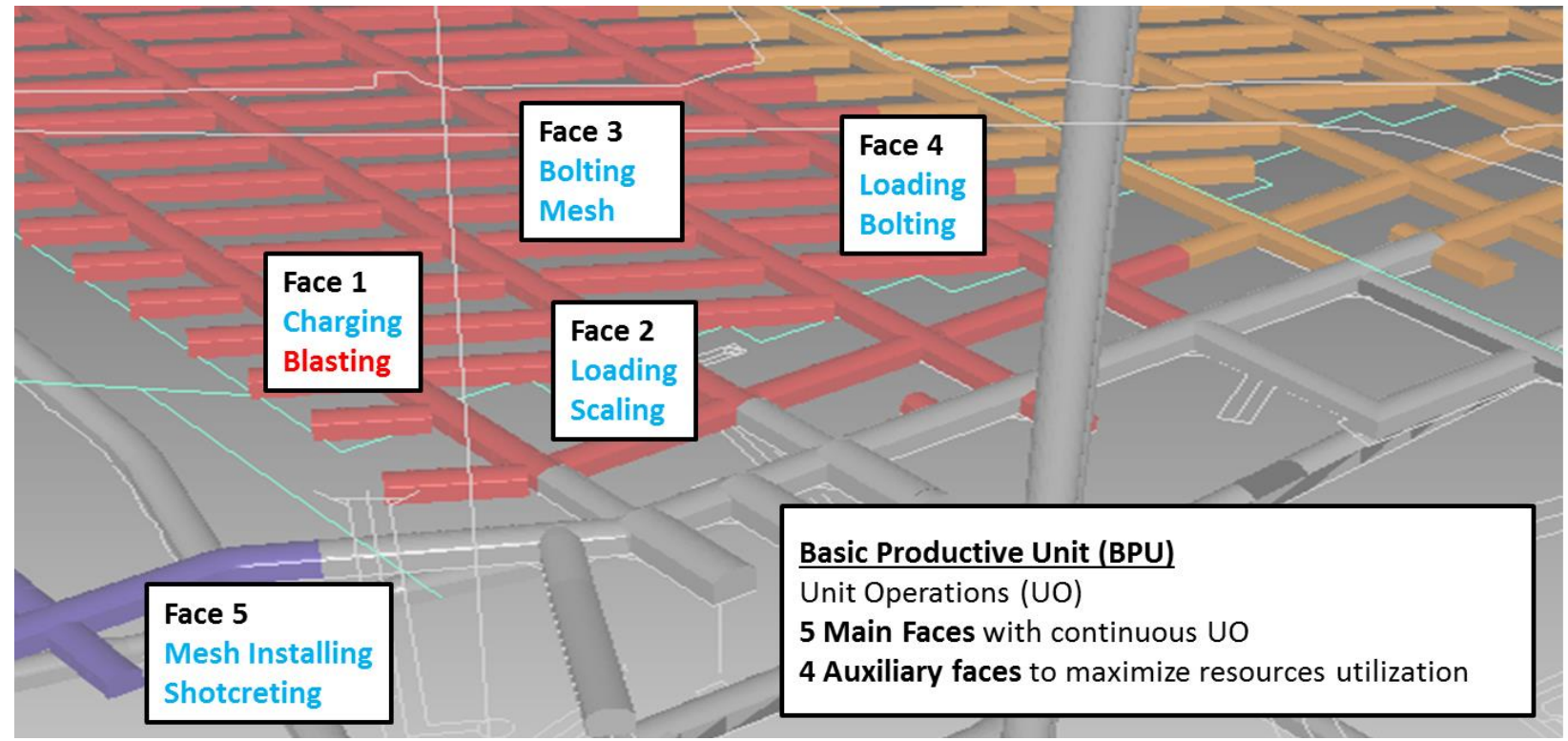

Figure 2 Scheme of BPU

Each BPU requires the minimum amount of faces to reach its full capacity (five main faces and four auxiliary faces). Theses faces are selected in accordance with mine configuration, medium- and long-term strategies, and mine design. Since the case study has a block caving layout, BPU in the production level comprises production drifts (main development faces $32 \mathrm{~m}$ apart) and crosscuts (16 m apart). Therefore, travel distances of allocated faces only range from 150-250 m. A more complex situation involving greater travel distances than the case study described would result in BPU working below its full capacity, similar to when a BPU does not have the minimum amount of main and auxiliary faces.

Services required for BPU operation comprise of:

- Ventilation.

- Power.

- Industrial water.

- Compressed air.

- Drainage.

The proposed model assumes that industrial water, compressed air and drainage are available in required capacity and in time for each scheduled activity. On the other hand, ventilation and power are services dependent on allocated faces by BPU and even among BPUs (semi-independence) whereby a minimum amount is required to schedule BPU to its full capacity. Mucking is the activity that requires the most ventilation $(40,000 \mathrm{cfm})$, although, in multiple face operations, the requirement of the BPU is defined by the operation unit which is scheduled simultaneously.

The minimum requirement for the model is $127,000 \mathrm{cfm}$ for ventilation and $600 \mathrm{kVA}$ in terms of power.

The required equipment to operate BPU to its full capacity are:

- One jumbo (development).

- One jumbo (support).

- One roboshot (shotcrete sprayer).

- One scaler.

- Two telehandlers. 
Development crews are required for unit operations. The model considers crews of three miners which are allocated to grouting, bolting, meshing and charging activities.

With all previously indicated resources, the utilisation of at least five faces is ensured. It is necessary to consider conflicts and interactions that are part of the development cycle and, therefore, time must be added to the cycle of each face to account for:

- Advancement of services (industrial water, compressed air, drainage, ventilation duct) to the face.

- Mechanical availability of equipment.

With the proposed model, the requirements mentioned will yield the following development rates:

- Main faces: Fifteen blasts per month for each face or 75 blasts for the five main faces.

- Auxiliary faces: Five to six blasts per month for each face or 20 to 24 blasts for the four auxiliary faces.

- Total BPU: Development of 95 to 99 blasts per month or 342 to $356 \mathrm{~m}$ per month.

In summary, a full capacity BPU is able to ensure approximately $350 \mathrm{~m}$ per month (Figure 3 ) of development advance.

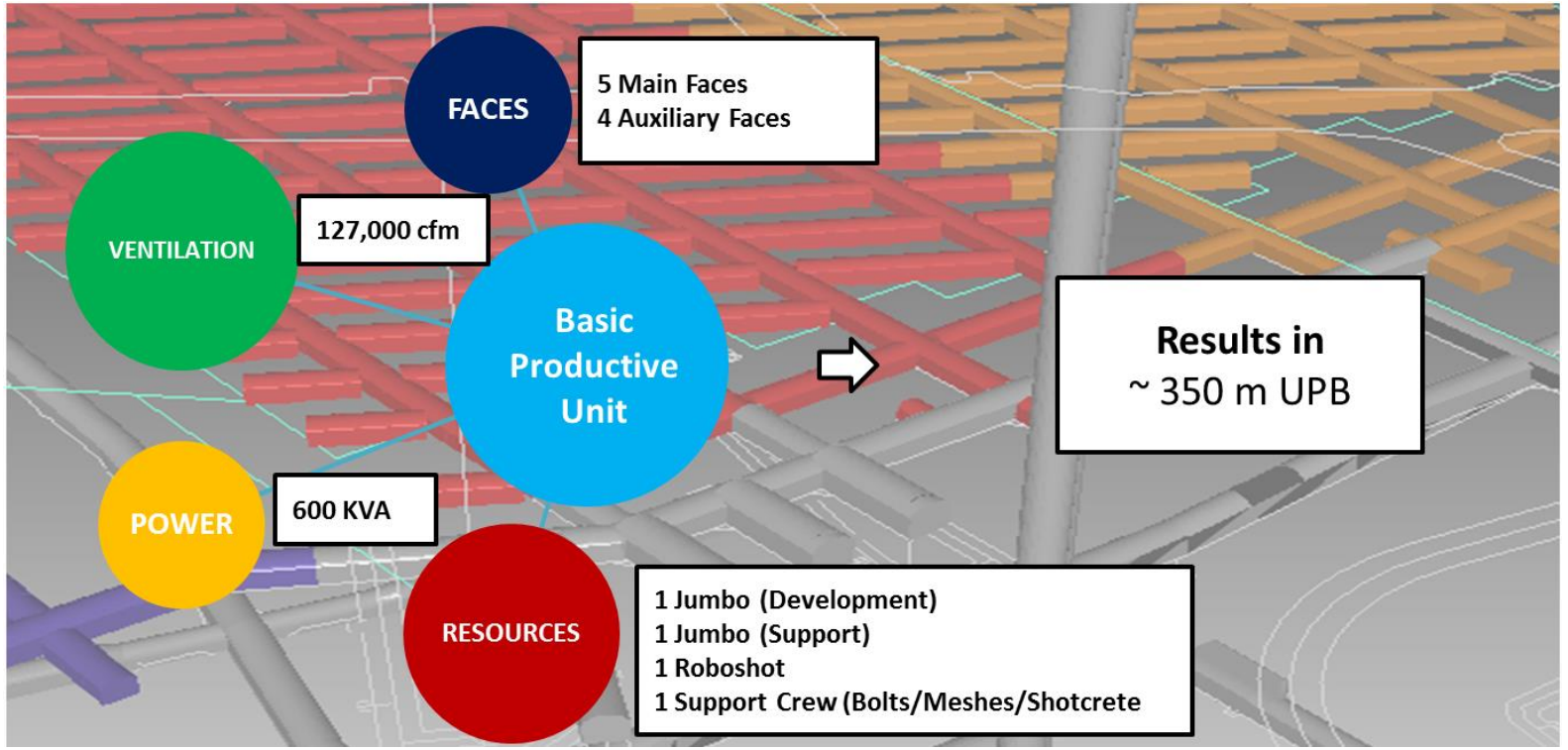

Figure 3 Performance of mining tunnels in terms of metres per month

\section{Case study}

The Chuquicamata Underground Project (Proyecto Mina Chuquicamata Subterránea, PMCHS) is a block caving project located in northern Chile, managed by Codelco. This project transitions the existing Chuquicamata open pit operation, the largest open pit in the world, to an underground mine, increasing its mine life to almost 100 years.

This transition is needed due to the size of the pit, increasing haulage distances and the increasing strip (waste/ore) ratio making the open pit operation unprofitable. In this scenario, the delivery of underground development milestones becomes a key factor to maintain production of Chuquicamata mine.

The project is being developed by three main contractors, each with a particular scope (Figure 4).

- CC-010 (Züblin): Production level and undercut level. Since development consists of a large number of faces (production drifts, crosscut, undercut drifts, etc.), the scheduling is mainly scheduled as a multiple-face scenario. 
- CC-013A (Astaldi): Ore-handling system infrastructure. The number of faces available is large enough for it to be considered as multiples faces, but tramming distances are greater than $250 \mathrm{~m}$. Strategy must mix between maximising advance by face and maximising utilisation of resources depending on priorities.

- CC-013B (Acciona Ossa): Main intake and exhaust tunnels. Mainly single-face development strategy. Completed in January 2018.

The project has reached a record of 3,600 m per month utilising these three main contractors, and in particular, CC-010 has reached a development rate of 1,700 to $1,800 \mathrm{~m}$ per month (around $50 \%$ of the project rate) due to multiple-face availability and the implementation of the proposed methodology.

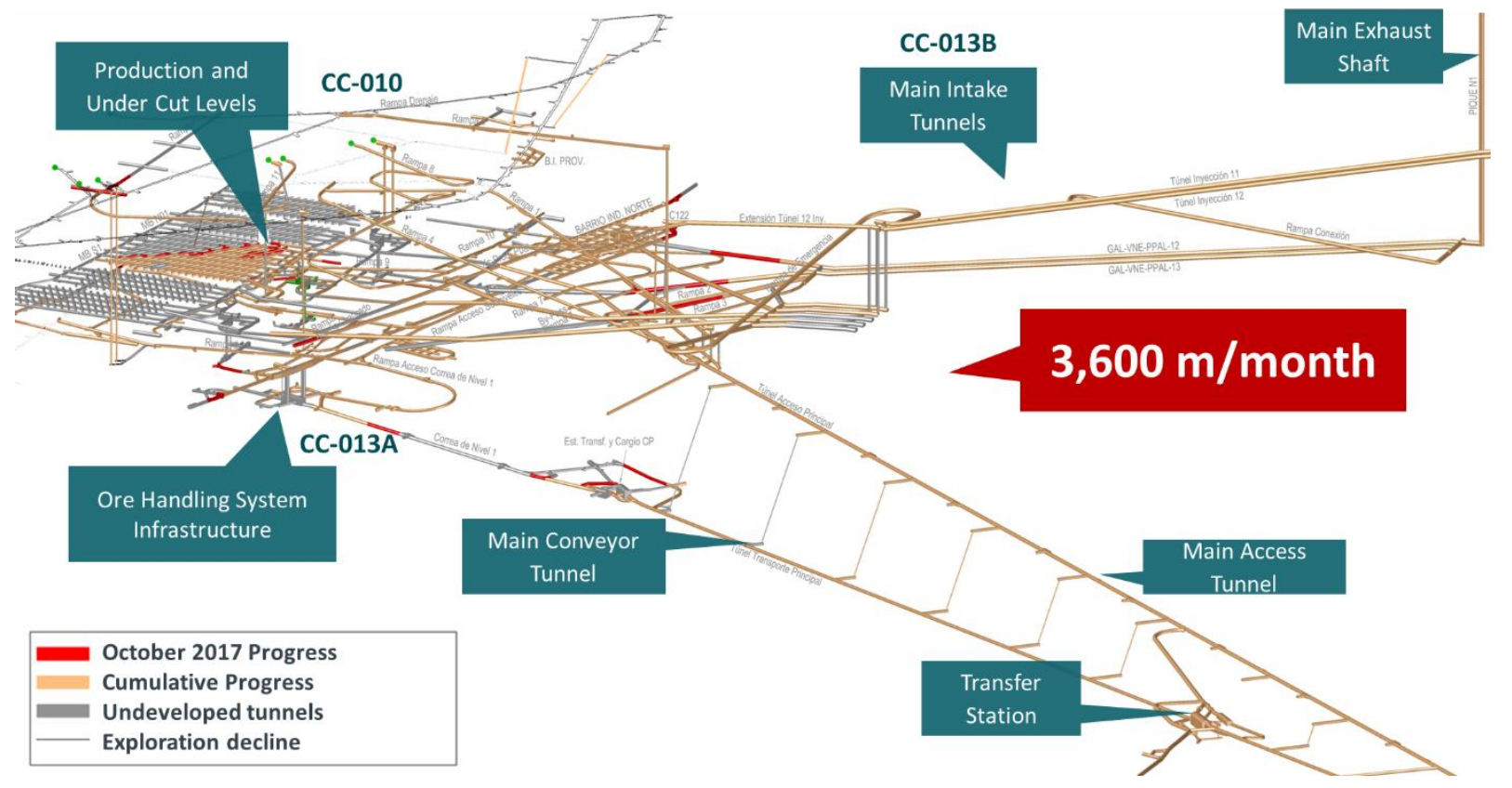

Figure 4 Main developments and contractors

In Figure 5, the development metres per month are shown from 2016 to March 2018. The proposed methodology was implemented in March 2017, increasing metres per month from 437 m in February 2017, prior to BPU, to $1,758 \mathrm{~m}$ in March 2018. The first six months of tunnel development without BPU (September 2016 to February 2017) have a clear trend, and thus a tunnel development of $512 \mathrm{~m}$ (an increase of 17\%) without BPU usage is extrapolated to March 2017. However, a tunnel development of $776 \mathrm{~m}$ was achieved in March 2017 (an increase of 78\%). Therefore, it can be concluded that the effect of BPU in its first month of implementation corresponds to a $61 \%$ increase in tunnel development rate, specifically, $264 \mathrm{~m}$ of the $339 \mathrm{~m}$ increase from February 2017 to March 2017.

The proposed methodology by itself is not the only factor that influenced the increase in rate of metres per month. It is necessary to match this scheduling approach with actions focused to ensure services for the defined BPU.

In the case study presented, it took nine months, from March to November 2017, to reach a rate greater than $1,700 \mathrm{~m}$ by month for the first time. This was accomplished by:

- Development of logistic and muck routes.

- Assurance of main services.

- Enablement of main access tunnel.

- Increase in blasting efficiency. 
All these factors ensure that the BPU was scheduled in its full capacity in terms of the number of blasts and the blasting efficiency of the cycle in order to increase the total amount of metres.

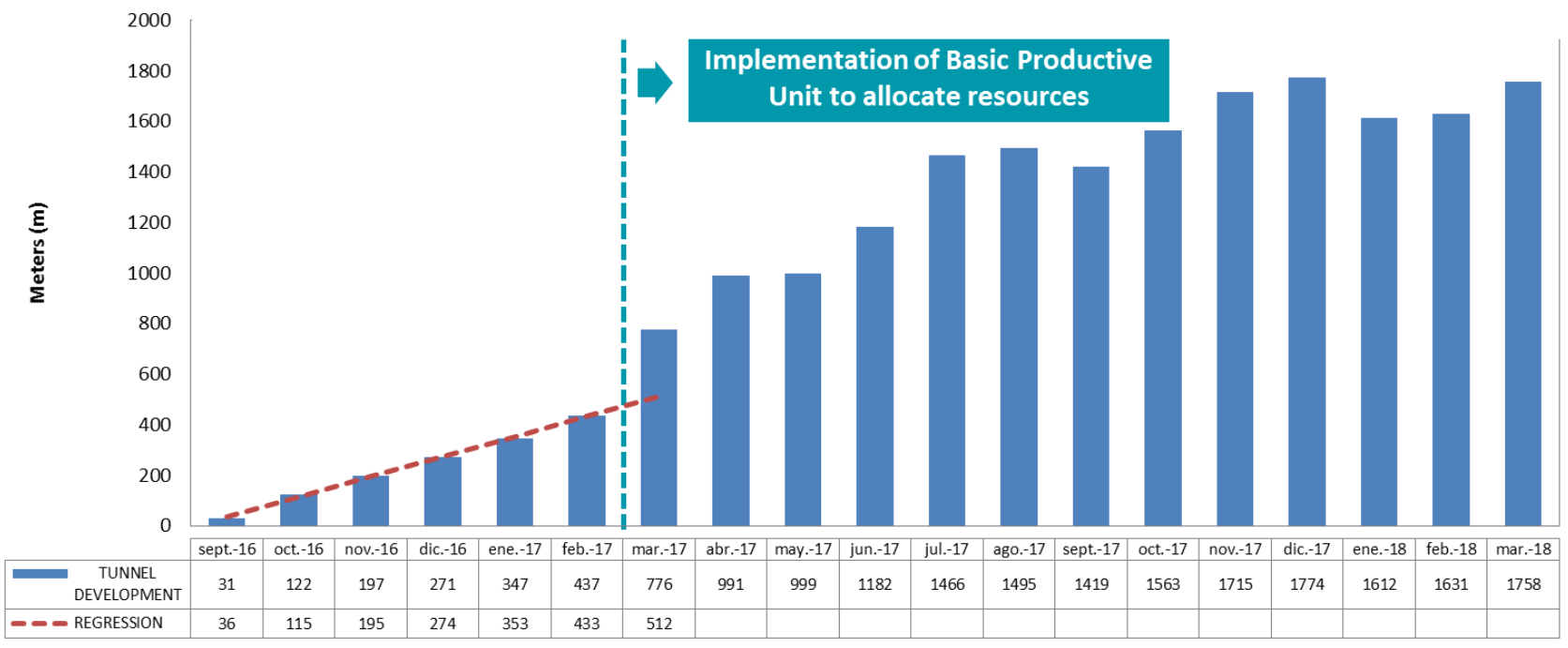

Figure 5 Performance of mining tunnels in terms of metres per month

The actual configuration of the CC-010 development allows the scheduling of six BPUs - two in the undercut level and four in the production level. Each BPU is linked to a development jumbo (Sandvik DD321 as shown in Table 1), assuming an availability of $85 \%$.

Table 1 Main CC-010 equipment

\begin{tabular}{lll}
\hline Equipment & Model & Total \\
\hline Development jumbo & Sandvik DD321 & 7 \\
Support jumbo (bolting) & Sandvik DD311 & 4 \\
Loader & Sandvik LH410 and LH514 & 8 \\
$20 \mathrm{t}$ truck & Atlas Copco MT2010 & 4 \\
$60 \mathrm{t}$ truck & Sandvik TH663 & 6 \\
\hline
\end{tabular}

The process of increasing development metres and capacity of each BPU involved a medium-term strategy that ensures requirements of the BPU approach. This medium-term strategy is updated every month in a three-month scope to ensure:

- Services: Ensuring at least 600 kVA of power and 127,000 cfm of ventilation for each BPU working at full capacity.

- Mucking strategy: Loading muck point and special routes for muck and logistics to isolate working areas and decrease interferences.

- Faces: Defining priority faces in the advance of the development focused on providing five main faces and four auxiliary faces per BPU. When this face availability criteria was achieved, the performance of each BPU began to reach its full capacity.

In Figure 6, the development scheduling using the BPU approach results in three units in the production level (BPU 2, 3 and 4), two units in the undercut level (BPU 5 and 6), and one unit involving both levels (BPU 1), with a total amount of $1,730 \mathrm{~m}$ scheduled in April 2018. The distribution of metres range from 167 to $411 \mathrm{~m}$ per BPU with an average value of $288 \mathrm{~m}$. 

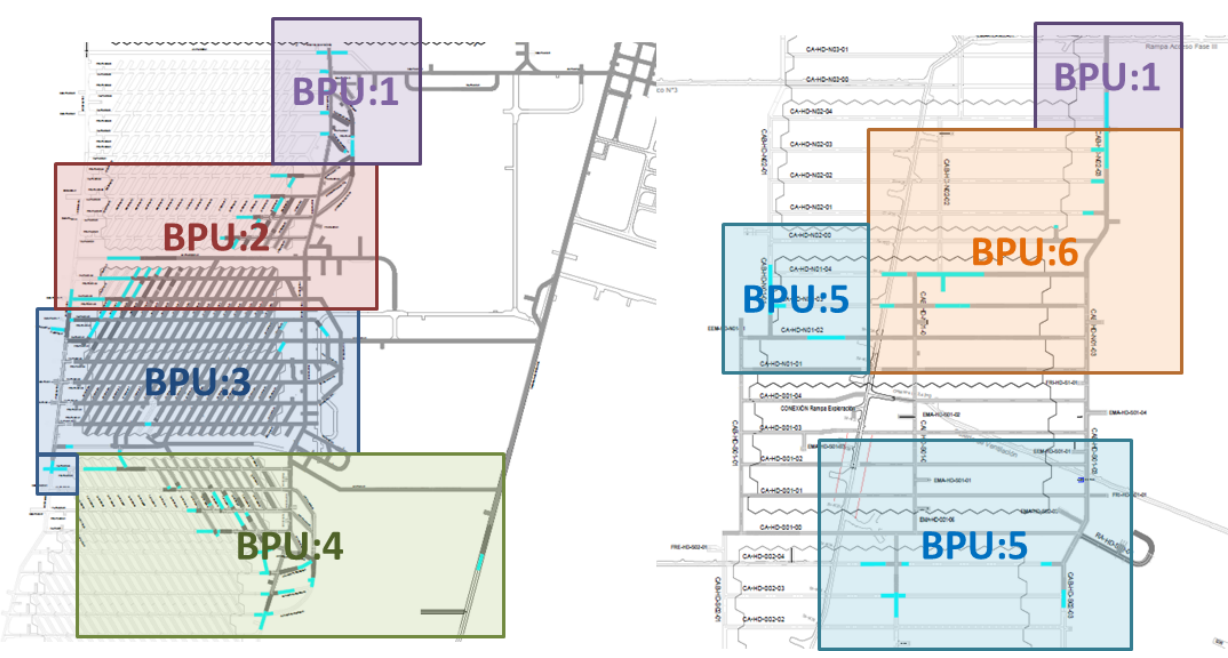

\begin{tabular}{|c|c|}
\hline \multicolumn{2}{|c|}{ Meters by BPU } \\
\hline BPU & Meters \\
\hline 1 & 167 \\
\hline 2 & 349 \\
\hline 3 & 359 \\
\hline 4 & 411 \\
\hline 5 & 209 \\
\hline 6 & 235 \\
\hline Total & $\mathbf{1 , 7 3 0}$ \\
\hline
\end{tabular}

Figure 6 BPU configuration in April 2018

In the case of BPUs in the production level, with a larger number of faces, metres ranged from 349 to $411 \mathrm{~m}$. BPU 1 had the smallest amount of metres scheduled $(167 \mathrm{~m})$, due to the number of faces and distance between them (it has faces both in the production level and undercut level).

In Figure 7, the metres per month per BPU is shown from June 2017 to March 2018, comparing the real performance and the scheduled rate. Only the multiple-face BPUs have been taken into account for the calculation. The results from the graph validate the proposed methodology, as the mean performance delivered is between 300 to $350 \mathrm{~m}$ per month.

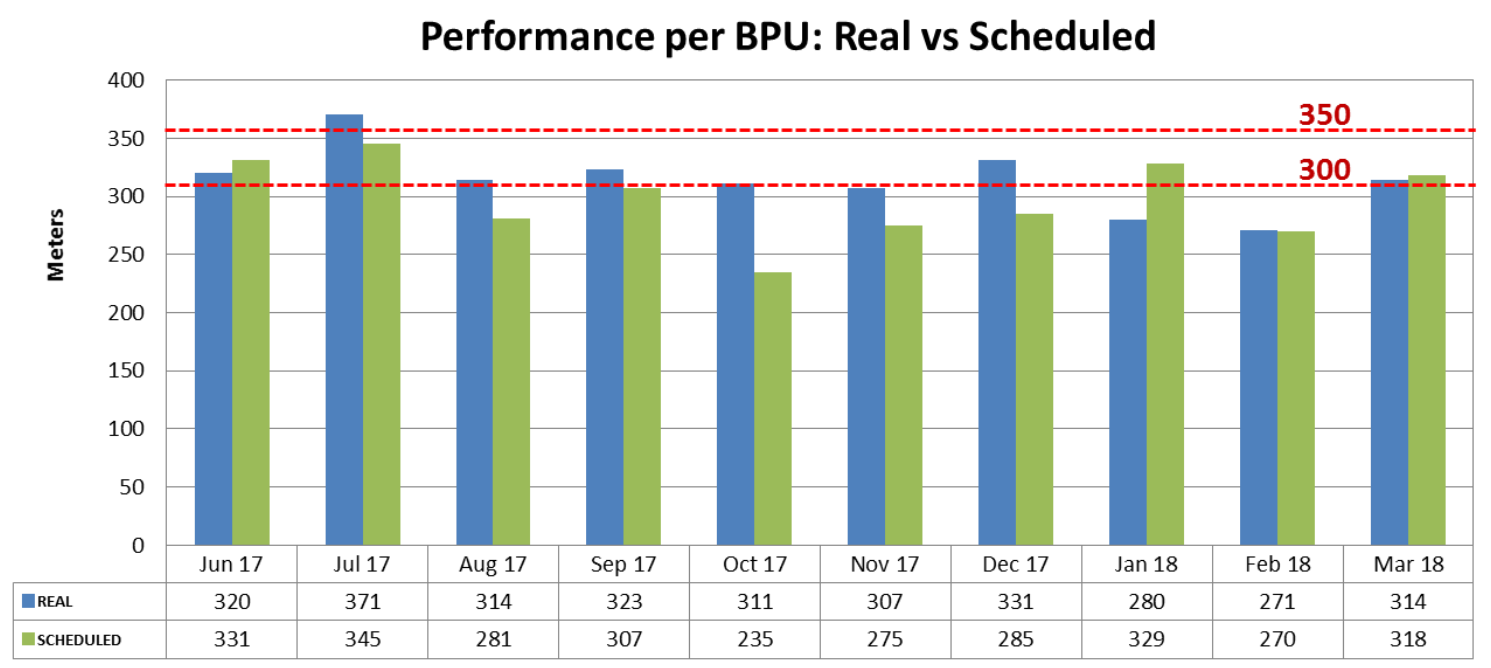

Figure 7 Performance per BPU by month

\section{Conclusion}

The proposed methodology can be easily implemented and updated. It offers a useful approach to monthly mining scheduling when multiple faces are available and being developed. This methodology ensures a rate ranging from 300 to 350 per month for each working area (BPU) and indicates the required conditions to accomplish this rate. 


\section{Acknowledgement}

The authors thank Codelco and Züblin for allowing us to publish the results from the implementation of the proposed approach in the Chuquicamata Underground Project.

\section{References}

Greberg, J \& Sundqvist, F 2011, 'Simulation as a tool for mine planning', Proceedings of the Second International Future Mining Conference, The Australasian Institute of Mining and Metallurgy, Melbourne, pp. 273-278.

Salama, A \& Greberg, J 2012, 'Optimization of truck-loader haulage system in an underground mine: a simulation approach using SimMine', Proceedings of MassMin 2012, Canadian Institute of Mining, Metallurgy and Petroleum, Westmount.

Wolgram, J, Li, Z \& Scoble M 2012, 'Application of simulation techniques in Oyu Tolgoi underground development scheduling', Proceedings of MassMin 2012, Canadian Institute of Mining, Metallurgy and Petroleum, Westmount. 